\title{
Habilidades dos enfermeiros no uso terapêutico do alteplase em unidade de pronto atendimento
}

RESUMO | Objetivo: avaliar as habilidades dos enfermeiros, no uso terapêutico do Alteplase, como terapia fibrinolítica, em pacientes com diagnóstico de infarto agudo do miocárdio. Método: A pesquisa foi realizada por meio de um estudo descritivo transversal, quantitativo, realizado por meio de questionário como instrumento de coleta, contendo 10 questões de múltipla escolha elaboradas pelo autor. A amostra foi constituída por 24 enfermeiros. A coleta de dados foi realizada em julho de 2019, com CAAE n 13159219.7.0000.5493. Os dados foram analisados e tratados por meio da análise descritiva. Resultado: Os resultados mostraram que os participantes da pesquisa possuem habilidades para o manuseio e aplicabilidade da terapia fibrinolítica, Alteplase, em pacientes acometidos por infarto agudo do miocárdio. Conclusão:Os resultados obtidos demonstram que os enfermeiros possuem habilidade para o manuseio, administração, aplicabilidade do Alteplase, bem como na avaliação dos sintomas e contraindicações do medicamento em pacientes acometidos por Infarto Agudo do Miocárdio. Entretanto, foi identificado uma porcentagem que apresentam dificuldades na execução de todas as atividades. Portanto, o estudo contribuirá na elaboração de protocolos aos profissionais da área da saúde envolvidos de modo direto ou indireto aos cuidados aos pacientes que necessitam desta intervenção farmacológica como tratamento.

Palavras-chaves: Enfermeiro; Terapia fibrinolítica; Alteplase; Infarto do miocárdio.

\begin{abstract}
Objective: to evaluate the abilities of nurses in the therapeutic use of Alteplase, as fibrinolytic therapy, in patients diagnosed with acute myocardial infarction. Method: The research was carried out by means of a transversal, quantitative descriptive study, carried out by means of a questionnaire as a collection instrument, containing 10 multiple choice questions elaborated by the author. The sample consisted of 24 nurses. The data collection was carried out in July 2019, with CAAE No. 13159219.7.0000.5493. The data were analyzed and treated through descriptive analysis. Result: The results showed that the research participants have skills for the handling and applicability of fibrinolytic therapy, Alteplase, in patients affected by acute myocardial infarction. Conclusion: The results show that the nurses have skills in the handling, administration and applicability of Alteplase, as well as in the evaluation of the symptoms and contraindications of the drug in patients affected by Acute Myocardial Infarction. However, it was identified a percentage that present difficulties in performing all activities. Therefore, the study will contribute in the elaboration of protocols to the professionals of the health area involved in a direct or indirect way to the care of patients who need this pharmacological intervention as treatment.
\end{abstract}

Keywords: Nurse; Fibrinolytic therapy; Alteplase; Myocardial infarction.

RESUMEN | Objetivo: evaluar las capacidades de las enfermeras en el uso terapéutico de la Alteplasa, como terapia fibrinolítica, en pacientes diagnosticados con infarto agudo de miocardio. Material y método: La investigación se realizó mediante un estudio descriptivo cuantitativo transversal, realizado mediante un cuestionario como instrumento de recopilación, que contenía 10 preguntas de opción múltiple preparadas por el autor. La muestra constaba de 24 enfermeras. La recopilación de datos se llevó a cabo en julio de 2019, con el CAAE No 13159219.7.0000.5493. Los datos fueron analizados y tratados mediante un análisis descriptivo. Resultado: Los resultados mostraron que los participantes en la investigación tienen habilidades en el manejo y la aplicabilidad del tratamiento fibrinolítico, Alteplase, en pacientes afectados por un infarto agudo de miocardio. Conclusión: Los resultados muestran que las enfermeras tienen aptitudes para el manejo, la administración y la aplicabilidad del Alteplase, así como para la evaluación de los síntomas y las contraindicaciones del fármaco en los pacientes afectados por un infarto agudo de miocardio. Sin embargo, se identificó un porcentaje que presenta dificultades para realizar todas las actividades. Por lo tanto, el estudio contribuirá a la elaboración de protocolos para los profesionales de la salud que participan directa o indirectamente en la atención de los pacientes que necesitan esta intervención farmacológica como tratamiento.

Palavras claves: Enfermera; Terapia fibrinolítica; Alteplasa; Infarto de miocardio.

\section{Lucio da Silva Ferreira}

Enfermeiro, Especialista em Docência para Ensino Superior em Enfermagem, Docente pelo Centro Universitário Anhanguera Vila Mariana. São Paulo -SP, Mestrando pela Universidade Anhanguera-Uniderp Pirituba, São Paulo- SP.

ORCID: 0000-0003-2381-0658

\section{Jefferson Carlos de Oliveira}

Enfermeiro, Mestrado Profissional em Enfermagem- Enfermagem no Processo de Cuidar em Saúde do Centro Universitário São Camilo, Docente do Curso de Enfermagem e Pós-Graduação em Urgência e Emergência - Centro Universitário Anhanguera de São Paulo Vila Mariana.

ORCID: 0000-0002-5258-7099

\section{Vania Claudia Olivo}

Farmacêutica, Mestre em Ciências Farmacêuticas pela Universidade de São Paulo (USP), Doutora em Ciências Universidade de São Paulo (USP), PósDoutorado em Farmacologia pela Universidade de São Paulo (USP), Professora adjunta pela Universidade AnhangueraUniderp Pirituba, São Paulo - SP. ORCID: 0000-0001-6689-0972

Recebido em: 12/09/2020

Aprovado em: 17/09/2020 
INTRODUÇÃO

$\Lambda$ s doenças cardiovasculares são as principais causas de morte no mundo. Anualmente, 17,1 milhões de pessoas são acometidas por essas doenças cardiovasculares e suas complicações, podendo evoluir para óbitoSendo importante ressaltar que, a organização Mundial da Saúde (OMS) presume que em 2030, a estimativa chegue a 23 milhões de óbitos por doenças cardiovasculares ${ }^{1}$.A grande prevalência dessas doenças pode ser explicada tanto pelo aumento da faixa etária da população quanto pela exposição desta a fatores de risco classicamente conhecidos como, hábitos alimentares inadequados, inatividade física, tabagismo, alcoolismo, aumento da prevalência da obesidade, estresse, além de fatores socioeconômicos ${ }^{2}$.

Dentre as doenças cardiovasculares, o Infarto Agudo do Miocárdio (IAM) representa um importante problema de saúde pública no mundo e no Brasil, apresentando altas taxas de incidência, prevalência e mortalida$\mathrm{de}^{2}$. Barbosa e cols (2019), citam que o IAM pode levar a óbito, anualmente, até 7,4 milhões de pessoas. ${ }^{2}$

$\mathrm{O}$ infarto agudo do miocárdio (IAM), atualmente, é considerado um importante problema de saúde pública no Brasil ${ }^{3}$.A maioria das mortes ocasionadas pelo IAM ocorrem nas primeiras horas de manifestação da doença, sendo $40 \%-65 \%$ dos casos na primeira hora e, aproximadamente, $80 \%$ nas primeiras 24 horas. Assim, a maior parte das mortes por IAM acontece fora do ambiente hospitalar, geralmente desassistidas por médicos. ${ }^{4}$

A melhor recuperação e, consequentemente redução dos índices de mortalidade do paciente pós IAM está associada à efetividade e a qualidade da assistência médica e de enfermagem. É primordial que a prevenção esteja associada a profissionais qualificados, aparelhos modernos para exames, instruções e informações
O infarto agudo do miocárdio (IAM), atualmente, é considerado um importante problema de saúde pública no Brasil.A maioria das mortes ocasionadas pelo IAM ocorrem nas primeiras horas de manifestação da doença, sendo 40\%-65\% dos casos na primeira hora e, aproximadamente, $80 \%$ nas primeiras 24 horas. Assim, a maior parte das mortes por IAM acontece fora do ambiente hospitalar, geralmente desassistidas por médicos. devidas ao paciente são fundamentais na redução do número de $\operatorname{casos}^{5}$. Nesse contexto, o enfermeiro é o profissional primordial na composição da equipe de saúdee responsável pela administração de medicamentos previamente estabelecidos em programas de saúde pública e em rotina aprovada pela instituição de saúde. Dentre esses medicamentos, particularmente, aqueles prioritários e de alto risco, participando de programas e atividades de educação sanitária, visando à melhoria de saúde do indivíduo, da família e da população em geral ${ }^{6}$.

Para o tratamento e reperfusão do miocárdio após o IAM, existem várias alternativas e segundo os resultados observados, as medicações intervencionistas garantem uma melhor sobrevida ao paciente, bem como uma maior preservação tecidual ${ }^{7}$. Os fármacos fibrinolíticos foram a primeira terapia utilizada para reperfusão para pacientes com IAM. Essa classe de medicamento ativa o sistema fibrinolítico endógeno e produz rápida redução ou resolução do trombo, restaurando o fluxo coronário, sendoa Estreptoquinase o primeiro fibrinolítico a demonstrar sua eficácia no tratamento do IAM. ${ }^{8}$ Os principais efeitos colaterais da estreptoquinase são hipotensão arterial, reações alérgicas e sangramentos. É importante salientar que, a readministração de fibrinolíticos em pacientes com falência da primeira dose não é recomendada, devido ao elevado risco de sangramento e a menor possibilidade de reperfusão na segunda tentativa. ${ }^{9}$

O agente fibrinolítico Alteplase, diferentemente da estreptoquinase, necessita de uma ligação com a fibrina para ser ativado e atuar na conversão do plasminogênio em plasmina, ou seja, é um ativador de plasminogênio tecidual humano recombinante, uma glicoproteína que ativa o plasminogênio diretamente para plasmina. Quando administrado por via endovenosa, permanece relativamente inativo no sistema circulatório. Uma vez ligada à fibrina, a substância é ativada, induzindo a conversão de plasminogênio 
em plasmina, que, por sua vez, promove a dissolução da fibrina do coágulo facilitando a circulação local. ${ }^{10,11}$

O tratamento fibrinolítico no infarto agudo do miocárdio, na embolia pulmonar aguda grave com instabilidade hemodinâmica, e no de acidente vascular cerebral isquêmico agudo sãoterapêuticas aprovados pela ANVISA para o uso do Alteplase. ${ }^{12}$ Portanto, é de suma importância que profissionais de saúde, sobretudo médicos e enfermeiros que irão realizar o procedimento de terapia fibrinolítica se mantenham sempre atualizados quanto aos mecanismos de ação desta medicação e de suas contra indicações. ${ }^{13}$ Diante do exposto, esse estudo teve como objetivo avaliar a habilidade dos enfermeiros no uso terapêutico de Alteplase, como terapia fibrinolítica intervencionista, suas indicações, contraindicações e reações adversas, bem como a forma de cuidados durante sua administração.

\section{MÉTODO}

Trata-se de uma pesquisa de caráter descritiva transversal, quantitativa, que foi realizada utilizandoum questionário como instrumento de coleta.O instrumento de pesquisa utilizado foi desenvolvido com as seguintes características:

a) Desenvolvido pelos autores, com base na experiência vivenciada na assistência em pacientes cardiológicos, fundamentando em diretrizes e protocolos.

b) Foram elaboradas três seções: Seção 1 - Dados Sociodemográficos dos participantes; Seção 2 - Sobre o IAM contendo seis questões de múltipla escolha, podendo o participante optar por mais de uma resposta para a mesma questão; Seção 3 - Sobre o uso terapêutico do Alteplase, composta por 10 questões de múltipla escolha, podendo o participante optar por mais de uma resposta para a mesma pergunta.

Em relação ao questionário, o mesmofoi composto por perguntas fechadas, sendo a disposição das respostas em alternativas, com o intuito de avaliar as habilidades dos enfermeiros no uso da terapia fibrinolítica, posologia, contraindicações, reações adversas e interações medicamentosas na utilização do Alteplase, tratando-se de questionário ainda não validado. A amostra de participantes foi composta pelas equipes de Enfermeiros da Unidade de Urgência e Emergência do Hospital da Zona Noroeste, Pronto-Socorro Central e Pronto-Socorro da Zona da Orla Intermediária, município

Figura 1 Distribuição dos profissionais por unidade de trabalho e a caracterização das variáveis socio demográfica dos participantes.

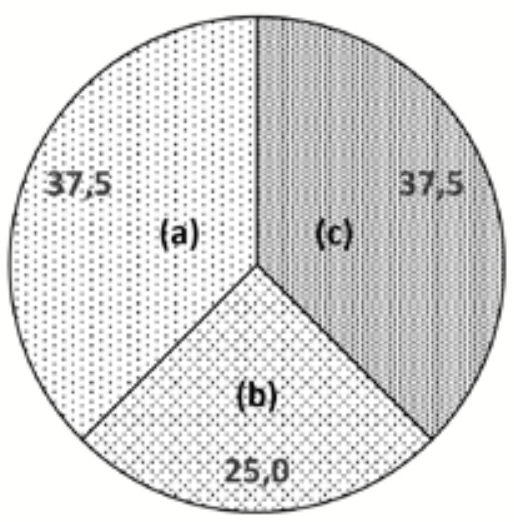

Onde a: enfermeiros da Unidade Zona Noroeste, b: de Hospital de Pequeno Porte e c: do Pronto Socorro ZOI. de Santos - SP, perfazendo 38 Enfermeiros lotados entre as três unidades. Após o convite e os critérios estabelecidos, a amostra foi constituída de 24 profissionais que optaram em participaram da pesquisa. A coleta de dados foi realizada no mês de julho de 2019. Considerou-se como critérios de inclusão, ser enfermeiro, membro permanente do quadro de funcionário do referido hospital e que tenha concordado em participar da pesquisa. Os critérios de exclusão, estabelecidos foram enfermeiros que durante o período de coleta estivessem gozando de férias ou de licença e os que não desejassem participar da pesquisa. A análise imparcial dos dados levantados e a preservação da identidade, foram identificados da seguinte maneira: a) enfermeiros lotados no Pronto Socorro da Zona Noroeste: EZN 1, EZN 2, EZN 3 ; b) enfermeiros lotados no Pronto Socorro Central: EPC 1, EPC 2, EPC3; c) enfermeiros lotados no Pronto Socorro da Zona da Orla Intermediária: EZOI 1, EZOI 2, EZOI 3. A análise dos resultados foi por meio do software Epi InfoT$M$ (acesso livre) que auxilia na análise de material qualitativo, com as ferramentas de codificação e armazenamento de textos em categorias específicas.Os dados obtidos através do software, foram compilados para o programa Excel () versão 1902 do Microsoft Office 365 para serem tabulados e posteriormente realizada a análise descritiva das variáveis. O presente estudo foi submetido a Plataforma Brasil e ao Comitê de Ética e Pesquisa da Universidade Anhanguera Campus Pirituba, com parecer favorável sob o CAAE n ${ }^{\circ}$ 13159219.7.0000.5493, e parecer 3.507.979.

\section{RESULTADOS}

A amostra foi constituída por 24 profissionais de enfermagem, sendo $37,5 \%$ alocados na Unidade Zona Noroeste, $25 \%$ no Hospital de pequeno porte e $37,5 \%$ na Unidade do Pronto Socorro ZOI. 
Tabela 1: Dados sociodemográficos dos participantes das Unidades Zona Noroeste, Hospital de Pequeno Porte e do Pronto Socorro ZOI. Santos- 2020.

\section{Variável}

Gênero

Masculino

Feminino

Raça

\begin{tabular}{llc} 
Branco & 17 & 70,84 \\
\hline Negro & 03 & 12,50 \\
\hline Pardo & 04 & 16,66
\end{tabular}

Idade

$\begin{array}{lll}31-40 \text { anos } & 10 & 42,00 \\ 41-50 \text { anos } & 06 & 25,00 \\ 51-69 \text { anos } & 08 & 33,00\end{array}$

Tempo de experiência na função

$\begin{array}{llc}6 \text { a } 10 \text { anos } & 05 & 20,84 \\ 10 \text { a } 15 \text { anos } & 08 & 33,33 \\ 15 \text { a } 20 \text { anos } & 02 & 8,33 \\ >20 \text { anos } & 09 & 37,50\end{array}$

\section{Grau de Instrução}

Especialização

\section{Mestrado}

Doutorado

20

83,34

\section{Tempo de conclusão Especialização}

\begin{tabular}{lcc}
1 a 5 anos & 02 & 9,09 \\
6 a 10 anos & 12 & 54,54 \\
11 a 15 anos & 03 & 13,64 \\
16 a 20 anos & 01 & 4,55 \\
\hline de 21 anos & 04 & 18,18 \\
FONTE: Dados de pesquisa, 2020. & &
\end{tabular}

Tabela 2:Autopercepção do enfermeiro sobrea habilidade em reconhecer o Infarto Agudo do Miocárdio (IAM), Santos- 2020.

\begin{tabular}{llc} 
Variável & N & $\%$ \\
\hline Caracterização do IAM & & \\
\hline Obstrução total da artéria por trombo & 24 & 100 \\
\hline Obstrução parcial da artéria por trombo & 13 & 54,16 \\
\hline Espasmo arterial & 06 & 25,00 \\
\hline Sintomas do IAM & & \\
\hline Dor precordial irradiada para o braço esquerdo & 14 & 58,34 \\
\hline Dor precordial irradiada para o braço direito & 03 & 12,50 \\
\hline Dor epigástrica & 12 & 50,00 \\
\hline
\end{tabular}

A Figura 1 mostra a distribuição dos profissionais por unidade de trabalho.

A Tabela 2 descreve os dados relativos a habilidade do enfermeiro em reconhecer o Infarto Agudo do Miocárdio (IAM).

A respeito da habilidade do enfermeiro na utilização da terapia fibrinolítica, os dados são descritos conforme a Tabela 3.

\section{DISCUSSÃO}

Os resultados apresentadosapontam que a idade média dos participantes foide 45 anos, $70,84 \%$ se declararam brancos e $87,50 \%$ eram do gênero feminino. Em relação ao tempo de experiência na função, 37,5\% declararam ter mais de 20 anos. Quando questionados sobre as suas habilidades em reconhecer o IAM, foi possível observar que $100 \%$ dos profissionais de enfermagem responderam que os pacientes apresentam uma obstrução total de uma artéria coronariana por trombo, sendo observado eventos, como a obstrução parcial da artéria e o espasmo arterial. Os benefícios oriundos pelo uso dos fibrinolíticos em pacientes sob tratamento do IAM nas primeiras horas, demonstram a relação entre a preservação da função ventricular e concomitante a redução de mortalidade ${ }^{14}$.O estudo corrobora com os dados apresentados pelo estudo de Gusto II B, publicado na The New Englandjournalof Medicine onde se empregou o uso de alteplase, resultando em redução de dez mortes adicionais por mil pacientes tratados ${ }^{15}$.

Adicionalmente, quando questionados sobre os sintomas $58 \%$ responderam que os pacientes apresentam dor torácica irradiada para o braço esquerdo e $50 \%$ assinalaram que os pacientes podem apresentar dor epigástrica intensa irradiada para região dorsal. Cerca de $37 \%$ dos participantes responderam que o paciente pode não apresentar dor. Com relação ao tempo dos sintomas, $87,50 \%$ declaram que a dor pode ter início súbito, 83,33\% que pode ter iniciado há algumas horas, 45,83\% há 


\begin{tabular}{llc}
\hline $\begin{array}{l}\text { Dor precordial pode irradiar para braço esquerdo, tórax podendo ser confun- } \\
\text { dida com dor epigástrica }\end{array}$ & 24 & 100,00 \\
\hline Paciente pode relatar ausência de dor & 09 & 37,50 \\
\hline Quanto tempo de dor torácica & 21 & 87,50 \\
\hline Início súbito & 20 & 83,33 \\
\hline Início há algumas horas & 11 & 45,83 \\
\hline Início há alguns dias & 08 & 33,33 \\
\hline Início há algumas semanas & 12 & 50,00 \\
\hline Dor intermitente & & \\
\hline Alterações do Ecocardiograma (ECG) & 02 & 8,33 \\
\hline Supredesnivelamento de ST em apenas uma derivação & 19 & 79,17 \\
\hline Supredesnivelamento de ST em duas ou mais derivações contíguas & 01 & 4,17 \\
\hline Infradesnivelamento de segmento ST em apenas uma derivação & 01 & 4,17 \\
\hline Infradesnivelamento de segmento ST em duas ou mais derivações contíguas & 02 & 8,33 \\
\hline Complexo QRS alargado ou com detalhes e onda T invertida & 03 & 12,50 \\
\hline Não sabe & & \\
\hline Tempo para realização do ECG após a admissão da unidade de assistência & 23 & 95,83 \\
\hline 10 minutos & 01 & 4,17 \\
\hline 30 minutos & & \\
\hline Exames Laboratoriais & 04 & 16,67 \\
\hline Ureia, Sódio, Potássio, Troponina, Creatinina & 03 & 12,50 \\
\hline CPK, CK-MM, CK-MB, Creatinina, Uréia & 05 & 20,83 \\
\hline Hemograma, CPK, Troponina, Potássio, Sódio & 24 & 100,00 \\
\hline CPK, CK-MB, Troponina, CK-MM & 03 & 12,50 \\
\hline TGO, GGT, CPK, CK-MB & 01,17 \\
\hline Nãosabe & & 4 \\
\hline Fon & & \\
\hline
\end{tabular}

FONTE: Dados de pesquisa, 2020.

Tabela 3: Autopercepção do enfermeiro sobre sua habilidade a respeito do uso do Alteplase, Santos- 2020.

\begin{tabular}{|c|c|c|}
\hline Variável & $\mathrm{N}$ & $\%$ \\
\hline \multicolumn{3}{|c|}{ Pode-se afirmar que a medicação poderá ser diluída em soro fisiológico? } \\
\hline Sim & 12 & 50,00 \\
\hline Não & 09 & 37,50 \\
\hline Não sabe & 03 & 12,50 \\
\hline \multicolumn{3}{|c|}{$\begin{array}{l}\text { O Alteplase pode ser administrado de forma concomitante com ou- } \\
\text { tras medicações, no mesmo frasco de infusão e/ou no mesmo acesso } \\
\text { venoso de outras drogas? }\end{array}$} \\
\hline Sim & 0 & 0,00 \\
\hline Não & 22 & 91,70 \\
\hline Não sabe & 02 & 8,30 \\
\hline
\end{tabular}

alguns dias, 33,3\% há semanas e 50\% pode ser uma dor intermitente.

Assim, o tempo é um fator crucial e determinante que visa o enfermeiro juntamente com a equipe atuar visando estabelecer o manejo com rapidez, eficiência e agilidade no atendimento indo de encontro com os dados apresentados quanto ao reconhecimento dos sintomas apresentados pelos pacientes ${ }^{16}$.

Outro ponto relevante é o eletrocardiograma (ECG) que poderá apresentar alterações, sendo que $80 \%$ dos participantes relataram que o exame demonstra supradesnivelamento do segmento ST em duas ou mais derivação contíguas e $12,50 \%$ relataram não saber as alterações que o ECG pode apresentar. Costumeiramente são realizados os exames laboratoriais para auxiliar no diagnóstico do paciente com IAM, dentre eles, CPK, CK$-M B$, Troponina, CK-MM ${ }^{17}$.

Os achados quanto ao diagnostico vão de encontro com a literatura que fundamenta o diagnóstico por meio da história atual da doença, no eletrocardiograma e nos resultados de exames laboratoriais, sendo o prognostico de acordo com a gravidade da obstrução arterial coronariana da lesão miocárdica ${ }^{18}$.Após a entrevista, os resultados demonstram que $100 \%$ dos entrevistados relataram ter conhecimento dessa conduta.

O tempo de atendimento a um paciente com suspeita de IAM é muito importante e cerca de $95 \%$ dos entrevistados demonstraram que é essencial o atendimento em até 10 minutos após a sua ocorrência. O risco de hemorragia é muito grave e a suspeita é uma contraindicação absoluta. Cerca de $87 \%$ dos entrevistados demonstraram que estão cientes dessa conduta.

O paciente com IAM necessita receber suporte clínico com terapia trombolítica ou angioplastia percutânea a fim de reduzir a lesão permanente do musculo do miocárdio ${ }^{19}$. Adicionalmente, o tempo após o início dos sintomas também podem contribuir para o sucesso da terapia fibrinolítica. Cerca de 16\% dos partici- 


\begin{tabular}{|c|c|c|}
\hline Sim & 15 & 62,50 \\
\hline Não & 06 & 25,00 \\
\hline Não sabe & 03 & 12,50 \\
\hline \multicolumn{3}{|c|}{$\begin{array}{l}\text { Qual o limite máximo de tempo após a ocorrência do IAM o Altepla- } \\
\text { se poderá ser administrado? }\end{array}$} \\
\hline 06 horas & 19 & 79,16 \\
\hline 08 horas & 1 & 4,16 \\
\hline 12 horas & 04 & 16,68 \\
\hline \multicolumn{3}{|c|}{$\begin{array}{l}\text { Sintomas como sonolência, afasia, hemiparesia, convulsão podem } \\
\text { ocorrer durante o tratamento }\end{array}$} \\
\hline Sim & 22 & 91,67 \\
\hline Não & 0 & 0,00 \\
\hline Não sabe & 02 & 8,33 \\
\hline \multicolumn{3}{|l|}{ Contraindicações absolutas } \\
\hline Distúrbio hemorrágico significativo & 21 & 87,50 \\
\hline Histórico, evidência ou suspeita de hemorragia intracraniana & 16 & 66,67 \\
\hline Hipertensão grave & 08 & 33,33 \\
\hline Pancreatite aguda & 06 & 25,00 \\
\hline Neoplasia com alto risco de sangramento & 16 & 66,67 \\
\hline Hipersensibilidade ao medicamento & 13 & 54,17 \\
\hline Não sabe & 01 & 4,17 \\
\hline Conhecimento das advertências e precauções & TOTAL & $\%$ \\
\hline 0 látex da embalagem do medicamento pode causar alergia & 07 & 29,17 \\
\hline Múltiplas punções podem interferir no risco de hemorragia & 17 & 0,83 \\
\hline Paciente submetido ao RCP recente & 09 & 37,50 \\
\hline Possibilidade de arritmia e hipotensão grave & 18 & 75,00 \\
\hline Uso do Alteplase pode causar AVC hemorrágico & 08 & 33,33 \\
\hline Não sabe & 03 & 12,50 \\
\hline
\end{tabular}

pantes afirmaram que o limite de tempo de 12 horas após o início dos sintomas é o recomendado para a terapia. De acordo com Sallum e Paranhos, (2010), o enfermeiro ocupa lugar de destaque no atendimento aos pacientes com síndromes coronariana agudas, sendo primordial o conhecimento desta doença, a classificação e as alterações clínicas e terapêuticas além dos cuidados específicos no atendimento cardiológico ${ }^{20}$.

Sobre as advertências e precauções, $70,8 \%$ dos participantes relataram não saber que o látex da embalagem pode causar resposta alérgica do paciente, $75 \%$ afirmaram conhecer a possibilidade de ocorrer arritmia e hipotensão e 33\% relataram que tem conhecimento que o Alteplase pode causar acidente vascular encefálico hemorrágico.Sobre a preparação do medicamento para ser administrado, $37,50 \%$ dos participantes afirmaram conhecer a maneira correta e o veículo a ser utilizado (diluente específico). Cerca de $91 \%$ dos participantes relataram ter conhecimento que sonolência, hemiparesia, convulsões e afasia são sintomas que os pacientes podem apresentar durante o tratamento.De acordo com Knobel (2002) o enfermeiro diante do conhecimento e abordagem racional é capaz de determinar contribuições para o tratamento adequado e precoce minimizando complicações ${ }^{21}$.

Os resultados obtidos na pesquisavão de encontro com a literatura demonstrando que os enfermeiros possuem habilidades para o manuseio e aplicabilidade do medicamento, tendo como eixo norteador a elaboração de protocolos assistenciais para o cuidado de enfermagem mais efetivo ao paciente.

\section{CONCLUSÃO}

Dentre os resultados obtidos podemos destacar a necessidade do conhecimento do tempo para a administração, a forma de administração e as contraindicações do uso desta medicação. O conhecimento desses fatores é funda- 


\begin{tabular}{lcc|} 
Alterações hemodinâmicas & 23 & 95,84 \\
\hline Progressão da Insuficiência Cardíaca & 09 & 37,50 \\
\hline Hipotensão & 15 & 62,50 \\
\hline Dor torácica & 0 & 0,00 \\
\hline Não sabe & 0 & 0,00
\end{tabular}

tempo de internação, mortalidade e custo hospitalar.

Há a necessidade de padrões assistenciais que busquem a qualidade, eficácia e eficiência do trabalho da equipe.A educação continuada em saúde

Quanto ao tempo de infusão do Alteplase para o tratamento do IAM, qual o regime de administração deve ser seguido?

\begin{tabular}{|c|c|c|}
\hline $\begin{array}{l}15 \mathrm{mg} \text { em bolús IV, seguido de } 50 \mathrm{mg} \text { por } 30 \text { minutos, seguida de } 35 \mathrm{mg} \text { por } \\
60 \text { minutos, até a dosagem máxima de } 100 \mathrm{mg} \text { em } 90 \text { minutos. }\end{array}$ & 19 & 79,16 \\
\hline $\begin{array}{l}\text { Administração de } 10 \mathrm{mg} \text { em bolús IV, seguido de } 50 \mathrm{mg} \text { por } 60 \text { minutos, seguida } \\
\text { de } 10 \mathrm{mg} \text { por } 30 \text { minutos, até a dosagem máxima de } 100 \mathrm{mg} \text { em até } 3 \text { horas. }\end{array}$ & 02 & 8,33 \\
\hline Não sabe & 03 & 12,50 \\
\hline
\end{tabular}

FONTE: Dados de pesquisa, 2020.

mental, pois é o elemento-chave no tratamento dos pacientes com IAM na fase aguda e subaguda, estando pautado na prevenção de complicações, redução do deve estar presente em todas as ações, em todos os níveis de atenção para promover a saúde e prevenir doenças, implementando ideias e práticas que façam parte do cotidiano da população e que atenda às suas necessidades. $\mathrm{O}$ estudo realizado apresentou limitações importantes no que se refere ao tamanho da amostra, que ao se apresentar em um número reduzido, permitindo considerar os resultados encontrados apenas para a população em questão.

\section{Referências}

1. Barbosa NO, Santos MR, De Oliveira RW, Fernandes VS, Naziazeno SDS. Perfil de internamento de pacientes com Infarto Agudo do Miocárdio no Estado de Sergipe, dentre os anos de 2014 a 2018. $2^{\circ}$ Congresso Internacional de Enfermagem - $\mathrm{CIE} / 13^{\circ}$ Jornada de Enfermagem da Unit (JEU) -6 a 10 maio de 2019, v. 1, n. 1 (2019).

2. Vaz DWN, Rezende RWS, Barros TVL, Atta JFM, Santos Filho LC, Miranda TLKS. Descrição epidemiológica de pacientes hospitalizados com IAM no Estado do Pará (de 2015 a 2019). RevBra Edu Saúde. 2020, 10(1): 45-50.

3. Vaz J, Abelin AP, Schmidt MM, De Oliveira PP, Gottschall CAM, Rodrigues CG, De Quadros AS. Creation and implementation of a prospective and multicentric database of patients with Acute Myocardial Infarction: RIAM. ArqBrasCardiol. 2020; 114(3):446-455.

4. Avezum JRA, Feldman A, Carvalho AC, Sousa AC, Mansur ADEP, Bozza AE, et al. V Guideline of the Brazilian Society of Cardiology on acute myocardial infarction treatment with ST segment elevation. ArqBrasCardiol. 2015; 105(2 Suppl 1):1-105.

5. Prata Sobrinho JR, Carvalho, FLO, Pereira RSF, Chueh FR, Leal RA, Martins LRN, Gouveia VT, Dourado DSSO, Macêdo JRM, Matos JEJ, Alves RN. Infarto agudo do miocárdio: uma revisão bibliográfica. ScireSalutis. 2015; 5(2): 6-13.

6. Cofen. Decreto $\mathrm{n} 94.406 / 87$ de 30 de março de 1987. Regulamenta a Lei $n^{0} 7.498$, de 25 de junho de 1986, que dispõe sobre o exercício da Enfermagem, e dá outras providências. Disponível em 区http://www.cofen.gov.br/ decreto-n-9440687 4173.html区. Acesso em 20 abr 2020.

7. Crespo RC, Crespo EC, Sánchez GC, Barreto EC. Estreptoquinasa a casi 30 años de demostrada su eficacia en el infarto agudo de miocárdio. RevArchMed Camagüey. 2016; 20(1): 96-107.

8. Baruzzi ACA, Stefanini E, Manzo G. Fibrinolíticos: indicações e tratamento das complicações hemorrágicas. RevSocCardiol Estado de São Paulo. 2018; 28(4):421-427.

9. Baruzzi ACA, Stefanini E, Pispico A. Infarto agudo do miocárdio com supra de ST: trombólise em qualquer local que a medicação esteja disponível. RevSocCardiol Estado de São Paulo. 2018; 28(4):409-420.

10.GISSI-2: Gruppo Italiano per lo Studio dellaSopravvivenzanell'InfartoMiocardico. A factorial randomised trial of alteplase versus streptokinase and heparin versus no heparin among 12,490 patients with acute myocar- dial infarction. Lancet. 336(8707):65-71, 1990.

11. ISIS-2 (Second International Study of Infarct Survival) Collaborative Group. Randomised trial of intravenous streptokinase, oral aspirin, both, or neither among 17,187 cases of suspected acute myocardial infarction. Lancet. 13;2(8607):349-60, 1988.

12. Brasil. Nota Técnica $N^{\circ} 227 / 2013$ (atualizada em 27/11/2015). Informações sobre o uso do Alteplase. Disponível em

Vhttps://www.saude.gov.br/images/pdf/2016/janeiro/07/Alteplase--Atualizado-em-27-11-2015-.pdf区. Acesso 20 abr 2020.

13.Galiza DDF, De Moura OF, De Barros VL, De Luz GOA. Preparo e administração de medicamentos: erros cometidos pela equipe de enfermagem. RevBrasFarmHospServ Saúde São Paulo. 2014; 5(2): 45-50.

14. Mcphee SJ, Papadakis MA. Current Medical Diagnosis e Treatment. $50 U ̈$ ed.: Mc Graw Hill; 2011.

15. Randomized trial of intravenous heparin versus recombinant hirudin for acute coronary syndromes. The Global Use of Strategies to Open Occluded Coronary Arteries (GUSTO) Ila Investigators. Circulation. 1994 Oct; 90 (4): 1631-7.

16. Oliveira, Franciely, Wédja Monteiro da Silva, andGisleide Carvalho Góes Fernandes. "Percepção do enfermeiro sobre 0 atendimento ao paciente com suspeita de infarto agudo do miocárdio." Ensaios USF 1.1 (2017): 1-13. 17. Steg G, James SK, Atar D, Badano LP, Blömstrom-Lundqvist C, Borger MA, Di Mario C, Dickstein K, Ducrocq G, Fernandez-Aviles F, Gershlick AH, Giannuzzi $P$, Halvorsen $S$, Huber $K$, Juni $P$, Kastrati $A$, Knuuti J, Lenzen MJ, Mahaffey KW, Valgimigli M, van'tH of A, Widimsky P, Zahger D. ESC Guidelines for the management of acute myocardial infarction in patientspresentingwith ST-segmentelevation. Eur Heart J. 2012; 33: 2569-2619

18.0liveira LAM et al., Cuidados de Enfermagem ao paciente com infarto agudo do miocárdio: uma revisão integrativa. Brazilian Journal of Surgery and Clinical Research - BJSCR. 2019; v.28.

19. Santos JCA, Piaggi LFD. Percepção do enfermeiro sobre o atendimento ao paciente com suspeita de infarto agudo do miocárdio. Revista Mineira de Ciências da Saúde. 2012; 2v.

20.Sallum A. M; Paranhos W.Y. 0 Enfermeiro e as Situações de Emergência. 2. ed., São Paulo: Atheneu, 2010.

21. Knobel, E. Terapia intensiva: cardiologia. São Paulo: Atheneu, 2002. 